\title{
3-O-Glyceryl-2-O-hexyl Ascorbate Suppresses Melanogenesis through Activation of the Autophagy System
}

\author{
Yushi Katsuyama, ${ }^{* a}$ Norihisa Taira, ${ }^{a}$ Masato Yoshioka, ${ }^{a}$ Yuri Okano, ${ }^{b}$ and Hitoshi Masaki ${ }^{b}$ \\ ${ }^{a}$ SEIWA KASEI Co., Ltd.; 1-2-14 Nunoichicho, Higashiosaka, Osaka 579-8004, Japan: and ${ }^{b}$ School of Bioscience and \\ Biotechnology, Tokyo University of Technology; 1404-1 Katakuracho, Hachiouji, Tokyo 192-0982, Japan. \\ Received December 27, 2017; accepted February 20, 2018
}

The formation of skin pigmentation requires multiple steps, namely the activation of melanocytes, the synthesis of melanin, the transport of melanosomes to the tips of melanocyte dendrites and the transfer of melanosomes from melanocytes to surrounding keratinocytes. Recently, we reported that melanosomes accumulate in melanocytes when melanosome transport is disrupted and that they are then degraded by the autophagy system. In this study, we examined whether 3-O-glyceryl-2-O-hexyl ascorbate (VC-HG) suppresses melanogenesis through the activation of autophagy since VC-HG interferes with melanosome transport through the down-regulated expression of MyosinVa and Kinesin. The results demonstrate that VC-HGtreated B16 cells show an activation of autophagy through an increased expression level of Microtubule-associated protein 1 light chain 3 (LC3)-II and a decreased expression level of p62. Furthermore, the decrease of melanin content elicited by VC-HG was partially abolished by hydroxychloroquine or pepstatin A which are inhibitors of autophagy. Taken together, we conclude that VC-HG suppresses melanogenesis by activating the autophagy system.

Key words 3-O-glyceryl-2- $O$-hexyl ascorbate; melanosome transport; autophagy; microtubule-associated protein 1 light chain 3-II; p62

Chronic sun exposure alters the appearance of the skin and causes wrinkling, sagging and hyperpigmented spots due to acceleration of the skin aging process. ${ }^{1)}$ Among those effects, hyperpigmented spots such as solar lentigos appear on the skin surface due to excess melanin synthesis depending on the frequency of sun exposure. Melanin synthesis in melanosomes is catalyzed by tyrosinase, which is the rate-limiting enzyme, and by tyrosinase-related protein-1 (Tyrp1) and dopachrome tautomerase. ${ }^{2,3)}$ Melanosomes mature by accumulating melanin and are transported intracellularly from the perinuclear area to the tips of dendrites in melanocytes by interacting with several motor proteins such as Kinesin and MyosinVa. ${ }^{4}$ Finally, melanosomes are transferred to surrounding keratinocytes via globules and are then distributed throughout the skin. ${ }^{5)}$

In a recent study, we reported that mature melanosomes accumulate in melanocytes due to disruption of their transport to the tips of dendrites and are then degraded by the autophagy system. ${ }^{6}$ ) That result indicated that interfering with intracellular melanosome transport would be an effective approach to suppress or improve pigmentation since it may avoid the risk of hypopigmentation. In this study, we examined whether 3-O-glyceryl-2-O-hexyl ascorbate (VC-HG), which is an amphiphilic ascorbic acid derivative (Fig. 1), suppresses pigmentation by activating the autophagy system, since we previously demonstrated that VC-HG suppresses melanogenesis by interfering with intracellular melanosome transport. ${ }^{7)}$

\section{MATERIALS AND METHODS}

Materials VC-HG was synthesized and identified by SEIWA KASEI Co., Ltd. (Osaka, Japan). Dulbecco's modified Eagle's medium (DMEM) and synthetic melanin were purchased from Sigma-Aldrich (St. Louis, MO, U.S.A.). Fetal bovine serum (FBS) was purchased from Nichirei Bioscience (Tokyo, Japan). Theophylline was purchased from Wako Pure Chemical Industries, Ltd. (Osaka, Japan). Radio immunoprecipitation assay (RIPA) buffer, protease inhibitors and pepstatin A were purchased from Nacalai Tesque (Kyoto, Japan). The bicinchoninic acid (BCA) protein assay kit was purchased from Thermo Fisher Scientific (Waltham, CA, U.S.A.). Rabbit anti-Microtubule-associated protein 1 light chain 3 (LC3) and rabbit anti-p62 polyclonal antibodies were purchased from Cell Signaling Technology (Beverly, MA, U.S.A.). Donkey anti-rabbit immunoglobulin G (IgG) H\&L (Alexa Fluor 555) and donkey anti-goat IgG H\&L (Alexa Fluor 488) were purchased from Abcam (Cambridge, U.K.). The goat anti-Pmel17 polyclonal antibody was purchased from Santa Cruz Biotechnology (Santa Cruz, CA, U.S.A.). 4',6-Diamidino-2-phenylindole dihydrochloride (DAPI) was purchased from Dojindo Laboratories (Kumamoto, Japan).

Cell Culture B16 mouse melanoma 4A5 cells (B16 cells) were obtained from the Riken Cell bank (Ibaraki, Japan), and were cultured in DMEM with $10 \% \mathrm{FBS}$ at $37^{\circ} \mathrm{C}$ in a $5 \% \mathrm{CO}_{2}$

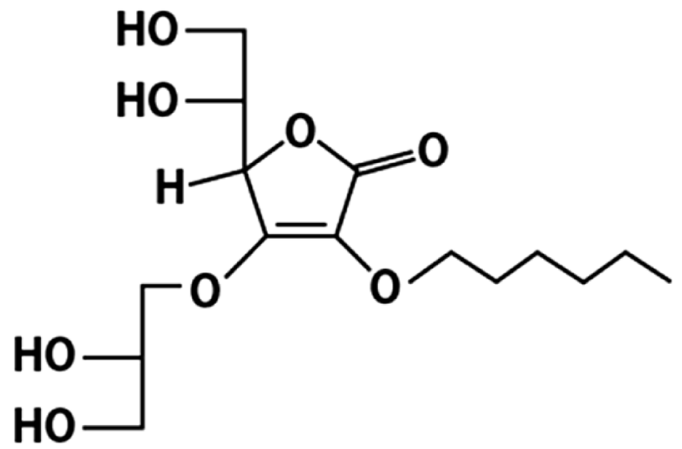

Fig. 1. The Structure of 3-O-Glyceryl-2-O-hexyl Ascorbate 
(a)

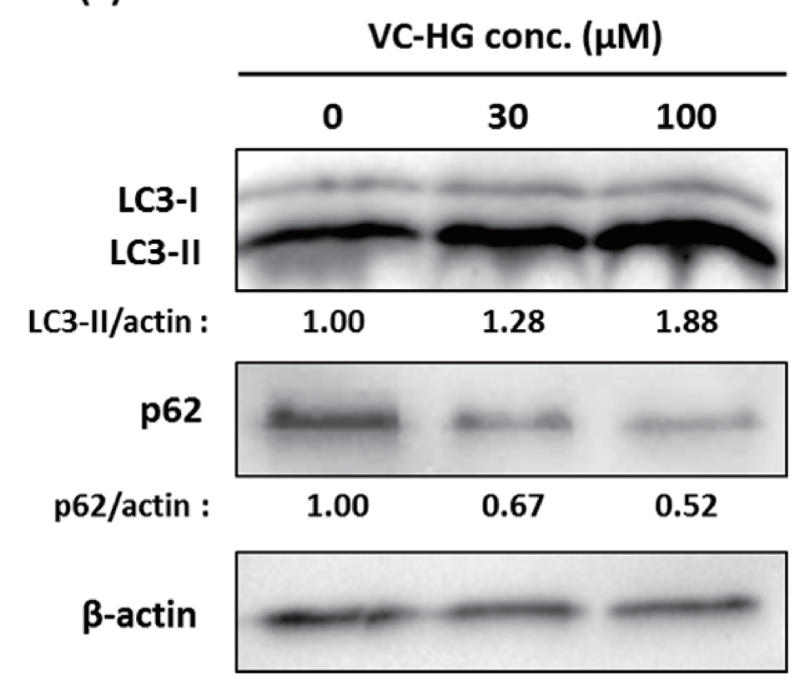

(b)

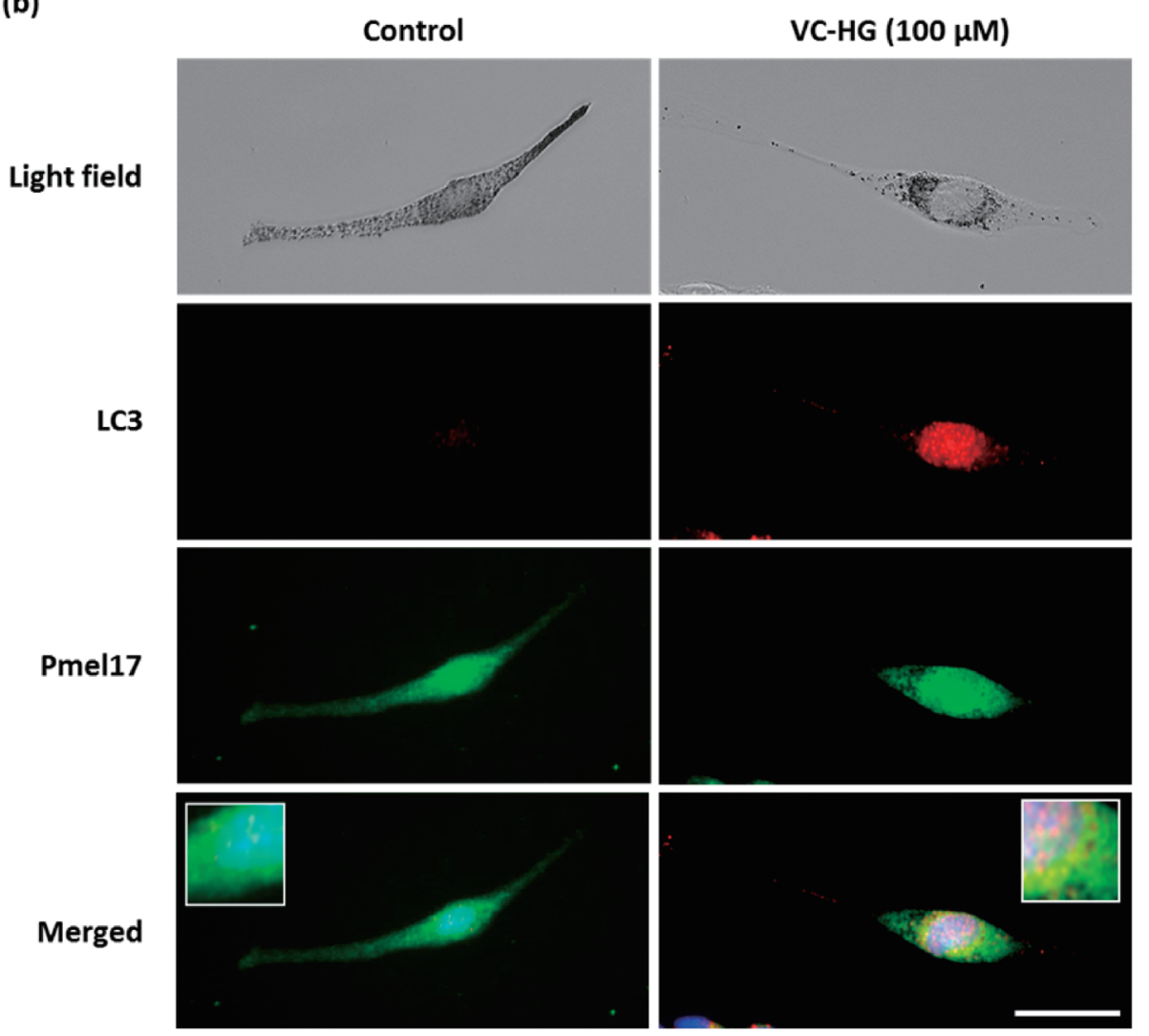

Fig. 2. Effect of Autophagy Activation by VC-HG in B16 Cells

After B16 cells were incubated with or without VC-HG at the concentrations noted for $72 \mathrm{~h}$ in the presence of theophylline, protein levels of LC3-I, LC3-II and p62 were measured by Western blotting (a); quantitation of bands noted under the blots were calculated versus the level of $\beta$-actin used as a loading control. Subcellular localization of LC3 and Pmel17 was detected by immunofluorescence analysis (b); cell nuclei are stained blue with DAPI. Bar scale: $50 \mu \mathrm{m}$.

atmosphere.

Western Blotting B16 cells $\left(1 \times 10^{5}\right.$ cells/well $)$ were seeded into 6-well plates in DMEM. After pre-culture with or without VC-HG in the presence of theophylline (1 mM) for $72 \mathrm{~h}$, cellular proteins were extracted using RIPA buffer with protease inhibitors. The solubilized proteins were separated using $10 \%$ or $15 \%$ sodium dodecyl sulfate-polyacrylamide gel electrophoresis (SDS-PAGE), and then were transferred to polyvinylidene difluoride membranes. The membranes were incubated with primary antibodies specific for LC3 (1:1000), p62 $(1: 1000)$ and $\beta$-actin $(1: 2000)$. Immunoreactive protein bands were then visualized using a WesternBreeze Chemiluminescent kit (Invitrogen, Carlsbad, CA, U.S.A.). Images were obtained and bands were quantitated using a ChemiDoc Touch Imaging System (Bio-Rad, Hercules, CA, U.S.A.).

Immunofluorescence $\mathrm{B} 16$ cells $\left(1 \times 10^{5}\right.$ cells/well $)$ were seeded into 6-well plates in DMEM. After pre-culture with or without VC-HG in the presence of theophylline (1 mM) for $72 \mathrm{~h}$, the cells were fixed with $4 \%$ formaldehyde, and then were permeabilized with $0.2 \%$ Triton X-100. After blocking 
(a)

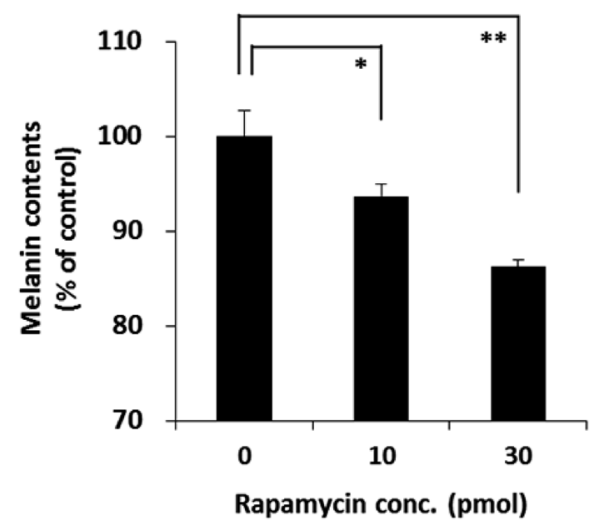

(b)

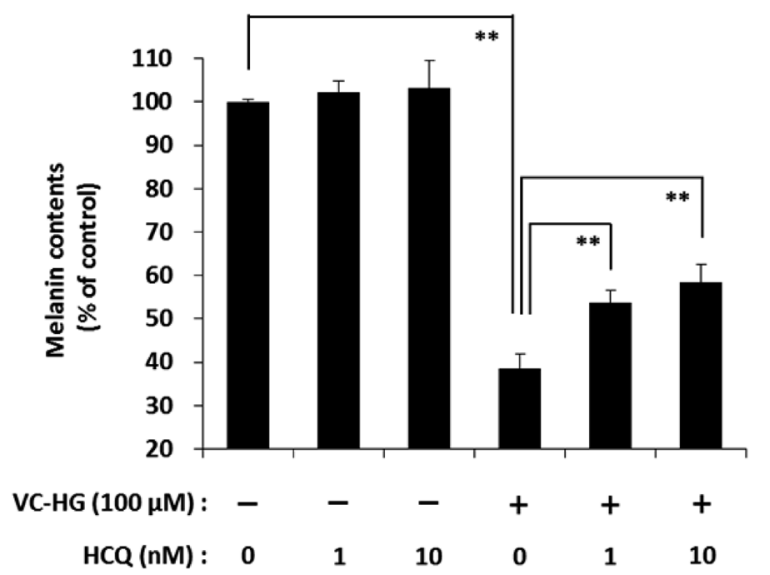

(c)

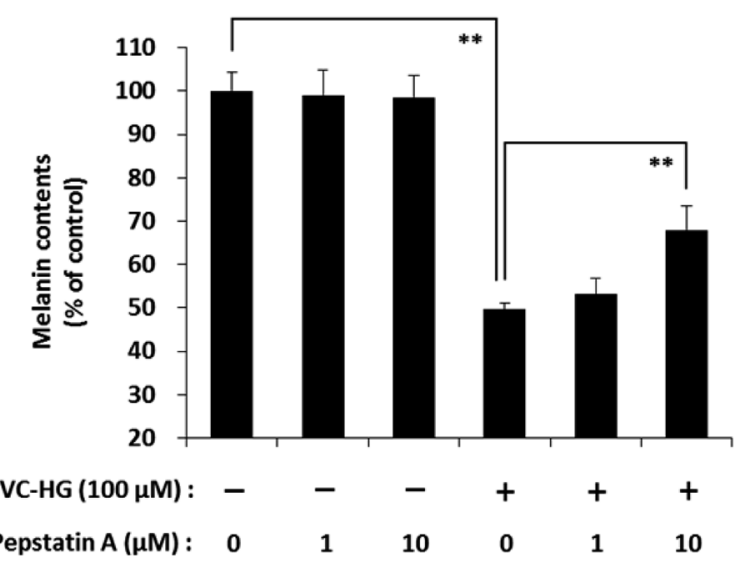

Fig. 3. Influence of Autophagy Activation by VC-HG on Melanogenesis

After B16 cells were incubated with or without rapamycin (a) or with or without VC-HG and/or HCQ (b) or pepstatin A (c) at the concentrations noted for $72 \mathrm{~h}$ in the presence of theophylline, intracellular melanin contents were measured. Bars indicate means \pm S.D. $(n=3)$. $* p<0.05$, ** $p<0.01$ indicate a significant difference between groups.

nonspecific binding with $1 \% \mathrm{IgG}$-free bovine serum albumin (BSA), $2 \mu \mathrm{g} / \mathrm{mL}$ rabbit anti-LC3 polyclonal antibody or goat anti-Pmel17 polyclonal antibody were used for immunofluorescent staining. Two micrograms per milliliter donkey anti-rabbit IgG H\&L (Alexa Fluor 555) or donkey anti-goat IgG H\&L (Alexa Fluor 488) were used to label antibodies that bound to LC3 and Pmel17. Cell nuclei were stained with DAPI. Confocal images were obtained using a BZ-X700 Fluorescence microscope (Keyence, Osaka, Japan).

Influence of Autophagy Activation by VC-HG on Melanogenesis $\mathrm{B} 16$ cells $\left(8 \times 10^{3}\right.$ cells/well $)$ were seeded into 48-well plates in DMEM. The cells were then incubated in the presence of theophylline for $72 \mathrm{~h}$ with or without $\mathrm{VC}-\mathrm{HG}$ and/or hydroxychloroquine (HCQ) and/or pepstatin A, which are inhibitors of autophagy that block the fusion of autophagosomes and lysosomes. ${ }^{8,9)}$ Intracellular melanin content was then quantified according to a previously described protocol. ${ }^{10)}$

Statistical Analysis All data are expressed as means standard deviation (S.D.) Comparisons between two groups were performed by Student's $t$-test. A $p$-value of less than 0.05 is considered statistically significant.

\section{RESULTS}

VC-HG Activates the Autophagy System The autophagy system is an intracellular clean-up system that maintains cellular homeostasis. ${ }^{11,12)}$ In that process, organelles and proteins that have lost their functions are trapped in autophagosomes containing phosphatidylethanolamine-conjugated microtubuleassociated protein $1 \mathrm{LC} 3-\mathrm{II}$, which is converted from LC3-I. ${ }^{13}$ ) p62 is involved in the formation of autophagosomes through its interactions with LC3-II and is also selectively degraded by autophagy as a substrate. ${ }^{14)}$ Hence, to characterize the potential role of $\mathrm{VC}-\mathrm{HG}$ on activation of the autophagy system, the behaviors of LC3-II and p62 were examined. We considered that a situation when melanosomes accumulated intracellularly would be better to examine effects of the autophagic system on melanosomes. Thus, treatment with theophylline, which stimulates melanin synthesis via the activation of cAMP signaling, ${ }^{15)}$ was used. B16 cells treated with VC-HG in the presence of theophylline showed an increase of LC3-II and a decrease of p62 in a dose-dependent manner (Fig. 2a). In a cytohistological study using immunofluorescence staining, B16 cells treated with VC-HG showed a higher expression level of LC3 than did untreated control cells (Fig. 2b). In addition, the co-localization of LC3 and Pmel17, which is a melanosome 
fibril protein, in the perinuclear area was observed (Fig. 2b).

VC-HG Suppresses Melanogenesis through Activation of the Autophagy System We first confirmed that B16 cells treated with rapamycin, which is an activator of autophagy via inactivation of mammalian target of rapamycin (mTOR), ${ }^{16}$ ) had a decreased intracellular melanin content (Fig. 3a). Interestingly, the decrease of intracellular melanin content in VC-HG-treated B16 cells in the presence of theophylline was significantly abrogated by treatment with HCQ or pepstatin A (Figs. 3b, c).

\section{DISCUSSION}

To date, chemicals used to treat hyperpigmentation of the skin have been developed focusing on the inhibition of tyrosinase activity. However, recent studies have reported that several chemicals used as anti-pigmentation agents have the risk of hypopigmentation due to reactions with tyrosinase that create toxic byproducts. ${ }^{17}$ ) Thus, interference with melanosome transport has recently been discussed as an effective alternate approach for anti-pigmentation to avoid the risk of hypopigmentation. It has been reported that melanosomes transferred from melanocytes to keratinocytes are degraded in keratinocytes by autophagy. ${ }^{18)}$ Also, we have reported that melanosomes that accumulate in melanocytes with disrupted transport are also degraded by autophagy. ${ }^{6)}$ The sum of these results suggests that degradation of melanosomes by the activation of autophagy would be an effective approach for inhibiting pigmentation. Our previous study reported that melanosomes accumulate in melanocytes due to interference with intracellular transport from the perinuclear region to the tips of dendrites and are then decomposed by the autophagy system. ${ }^{6}$ ) In addition, we demonstrated that VC-HG interferes with melanosome transport via the down-regulation of mRNA expression levels of transport-related proteins such as MyosinVa, Rab27a and Kinesin. ${ }^{7}$ Those results suggested that VC-HG activates the autophagy system. In this study, we found that VC-HG decreases melanin content in B16 cells (Figs. 3b, c). In order to clarify the relationship between intracellular melanin content and autophagy, we then examined the effects of rapamycin, which activates autophagy through the inhibition of mTOR, ${ }^{19)}$ on intracellular melanin content. Rapamycin reduced melanin content in cells in a dose-dependent manner, however that effect was not strong (Fig. 3a). Those results indicated that the autophagy system has a partial involvement in the modulation of intracellular melanin content. We then examined whether HCQ and/or pepstatin A, which are inhibitors of autophagy through their effects on lysosomes or lysosomal proteinases, respectively, restored the melanin content decrease elicited by VC-HG. HCQ and pepstatin A partially restored melanin content in a dose-dependent manner (Figs. 3b, c). These phenomena might be explained as anti-melanogenic characteristics of $\mathrm{VC}-\mathrm{HG}$, which markedly decreased the protein expression level of tyrosinase and interfered with melanosome transport. ${ }^{7)}$

Taken together, we conclude that VC-HG suppresses melanogenesis by activating the autophagy system and should be an effective approach to inhibiting skin pigmentation without the risk of hypopigmentation.

Conflict of Interest The authors declare no conflict of interest.

\section{REFERENCES}

1) Ichihashi M, Ando H, Yoshida M, Niki Y, Matsui M. Photoaging of the skin. Anti-Aging Med., 6, 46-59 (2009).

2) del Marmol V, Beermann F. Tyrosinase and related proteins in mammalian pigmentation. FEBS Lett., 381, 165-168 (1996).

3) Iozumi K, Hoganson GE, Pennella R, Everett MA, Fuller BB. Role of tyrosinase as the determinant of pigmentation in cultured human melanocytes. J. Invest. Dermatol., 100, 806-811 (1993).

4) Hume AN, Seabra MC. Melanosomes on the move: a model to understand organelle dynamics. Biochem. Soc. Trans., 39, 1191-1196 (2011).

5) Ando H, Niki Y, Ito M, Akiyama K, Matsui MS, Yarosh DB, Ichihashi M. Melanosomes are transferred from melanocytes to keratinocytes through the processes of packaging, release, uptake, and dispersion. J. Invest. Dermatol., 132, 1222-1229 (2012).

6) Katsuyama Y, Taira N, Yoshioka M, Okano Y, Masaki H. Disruption of melanosome transport in melanocytes treated with theophylline causes their degradation by autophagy. Biochem. Biophys. Res. Commun., 485, 126-130 (2017).

7) Taira N, Katsuyama Y, Yoshioka M, Okano Y, Masaki H. 3-OGlyceryl-2-O-hexyl ascorbate suppresses melanogenesis by interfering with intracellular melanosome transport and suppressing tyrosinase protein synthesis. J. Cosmet. Dermatol., (2017), in press.

8) Liu Q, Luo XY, Jiang H, Yang MH, Yuan GH, Tang Z, Wang H. Hydroxychloroquine facilitates autophagosome formation but not degradation to suppress the proliferation of cervical cancer $\mathrm{SiHa}$ cells. Oncol. Lett., 7, 1057-1062 (2014).

9) Barth S, Glick D, Macleod KF. Autophagy: assays and artifacts. $J$. Pathol., 221, 117-124 (2010).

10) Ninomiya K, Matsumoto $T$, Chaipech S, Miyake S, Katsuyama Y, Tsuboyama A, Pongpiriyadacha Y, Hayakawa T, Muraoka O, Morikawa T. Simultaneous quantitative analysis of 12 methoxyflavones with melanogenesis inhibitory activity from the rhizomes of Kaempferia parviflora. J. Nat. Med., 70, 179-189 (2016).

11) Mizushima N. Autophagy: process and function. Genes Dev., 21, 2861-2873 (2007).

12) Seglen PO, Bohley P. Autophagy and other vacuolar protein degradation mechanisms. Experientia, 48, 158-172 (1992).

13) Kabeya Y, Mizushima N, Ueno T, Yamamoto A, Kirisako T, Noda T, Kominami E, Ohsumi Y, Yoshimori T. LC3, a mammalian homologue of yeast Apg8p, is localized in autophagosome membranes after processing. EMBO J., 19, 5720-5728 (2000).

14) Sahani MH, Itakura E, Mizushima N. Expression of the autophagy substrate SQSTM1/p62 is restored during prolonged starvation depending on transcriptional upregulation and autophagy-derived amino acids. Autophagy, 10, 431-441 (2014).

15) Steinberg ML, Whittaker JR. Theophylline incorporation into the nucleic acids of theophylline-stimulated melanoma cells. J. Invest. Dermatol., 71, 250-256 (1978).

16) Wu L, Feng Z, Cui S, Hou K, Tang L, Zhou J, Cai G, Xie Y, Hong Q, Fu B, Chen X. Rapamycin upregulates autophagy by inhibiting the mTOR-ULK1 pathway, resulting in reduced podocyte injury. PLOS ONE, 8, e63799 (2013).

17) Nagata $T$, Ito $S$, Itoga $K$, Kanazawa $H$, Masaki $H$. The mechanism of melanocytes-specific cytotoxicity induced by phenol compounds having a prooxidant effect, relating to the appearance of leukoderma. Biomed. Res. Int., 2015, 479798 (2015).

18) Murase D, Hachiya A, Takano K, Hicks R, Visscher MO, Kitahara T, Hase T, Takema Y, Yoshimori T. Autophagy has a significant role in determining skin color by regulating melanosome degradation in keratinocytes. J. Invest. Dermatol., 133, 2416-2424 (2013).

19) Cai Z, Yan LJ. Rapamycin, autophagy, and Alzheimer's disease. $J$. Biochem. Pharmacol. Res., 1, 84-90 (2013). 